Z Rheumatol 2012 $\cdot 71: 550$

DOI 10.1007/s00393-012-0956-1

(c) Springer-Verlag 2012

I. Kötter ${ }^{1,2,3} \cdot$ A. Melms 4

${ }^{1}$ Universitätsklinikum Tübingen

${ }^{2}$ Abteilung für Allgemeine Innere Medizin und Nephrologie, Robert-Bosch-Krankenhaus, Stuttgart

${ }^{3}$ Rheumatologische Schwerpunktpraxis, Stuttgart Bad-Cannstatt

${ }^{4}$ Neurologische Rehabilitation, Neurologischen Klinik Universitätsklinikum Erlangen

\title{
Neurologie und Rheumatologie
}

ziert werden, wobei man bei Letzteren ohne eindeutige Kompressionswirkung keine zwingende Operationsindikation aussprechen würde.

\section{1) Neurologische Symptome sind bei den Arthritiden, insbesondere der rheumatoiden Arthritis häufig}

Wir haben uns in dem vorliegenden Leitthemenheft der Zeitschrift für Rheumatologie auf neurologische Begleitsymptome bei Kollagenosen und Vaskulitiden sowie Nebenwirkungen der modernen medikamentösen Therapie entzündlich rheumatischer Erkrankungen am Nervensystem konzentriert. Besondere Relevanz und aktuelle Bedeutung hat die Diskussion über das JC-Virus und die Manifestation einer progressiven multifokalen Leukenzephalopathie (PML) unter Immunsuppressiva und Biologika und das Auftreten demyelinisierender Erkrankungen bei TNF-Antagonisten. Bei Kollagenosen und Vaskulitiden sind die frühe Diagnose und eine intensivierte Therapie bei neurologischen Krankheitsmanifestationen entscheidend, da ein später Therapiebeginn die meist irreversiblen Schädigungen nicht mehr beeinflussen kann und eine erhebliche Beeinträchtigung der Lebensqualität verbleibt. Dies zeigt einmal mehr, wie wichtig eine gute interdisziplinäre Zusammenarbeit zwischen Rheumatologen und Kollegen anderer Fachdisziplinen, in diesem Fall mit den Neurologen, ist.

Wir freuen uns, dass es uns für dieses Leitthema gelungen ist, ausgewiesene Ex- perten für die Themen Kollagenosen, Vaskulitiden und medikamentöse Nebenwirkungen am Nervensystem zu gewinnen. Die Kapitel wurden jeweils von einem Autorenteam aus Vertretern beider Fachgebiete verfasst, um die Thematik aus unterschiedlichem Blickwinkel, aber dennoch aufeinander abgestimmt zu präsentieren. Ihre

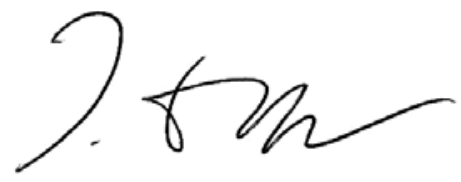

Prof. Dr. Ina Kötter

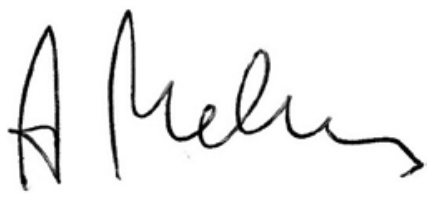

Prof. Dr. med. Arthur Melms

\section{Korrespondenzadressen}

\section{Prof. Dr. I. Kötter}

Abteilung für Allgemeine Innere Medizin und Nephrologie, Robert-Bosch-Krankenhaus Auerbachstr. 110, 70376 Stuttgart ina.koetter@med.uni-tuebingen.de

\section{Prof. Dr. A. Melms}

Neurologische Rehabilitation, Neurologischen Klinik Universitätsklinikum Erlangen

Schwabachanlage 6, 91054 Erlangen arthur.melms@uk-erlangen.de 